\title{
Organic Electronics for Point-of-Care Metabolite Monitoring
}

Anna-Maria Pappa ${ }^{1,2 *}$, Onur Parlak ${ }^{3 *}$, Gaetan Scheiblin ${ }^{4 *}$, Pascal Mailley $^{4}$, Alberto Salleo ${ }^{3}$ and Roisin M. Owens ${ }^{1,2}$

1. Department of Bioelectronics, École Nationale Supérieure des Mines, CMP-EMSE, MOC, 13541 Gardanne, France

2. Department of Chemical Engineering and Biotechnology, University of Cambridge,

Cambridge CB3 OAS

3. Department of Materials Science and Engineering, Stanford University, Stanford, California 94305

\section{CEA, LETI, MINATEC Campus, F-38054 Grenoble, France}

Correspondence: rmo37@cam.ac.uk (R. M. Owens)

Follow me on Twitter: biochemist_hero

*authors equal contribution

Keywords: organic electronics, metabolites, point-of-care, biosensors, wearable, diagnostics

\begin{abstract}
In this review we focus on demonstrating how organic electronic materials can solve key problems in biosensing thanks to their unique material properties and implementation in innovative device configurations. We highlight specific examples where these materials solve multiple issues related to complex sensing environments, and we benchmark these examples by comparing to state-of-the-art commercially available sensing using alternative technologies. We have categorized our examples by sample type, focusing on sensing from bodily fluids in vitro and on wearable sensors, which have attracted significant interest due to their integration with everyday life activities. We finish by describing
\end{abstract}


a future trend for in vivo, implantable sensors, which hopes to build on current progress from sensing in

biological fluids ex vivo.

\section{Glossary}

Amperometry measures the current of the working electrode set out of equilibrium at a fixed potential. Thus, when the substrate reaches the active site of the immobilized enzyme, electrons are rapidly exchanged.

Analyte is a compound (e.g. glucose, lactate, drug, pesticide) whose concentration is to be measured.

Biorecognition elements include biological components or derivatives that specifically interact with the analyte of interest (e.g. enzymes, organelles, cells, tissues, antibodies and nucleic acids)

Biosensor is an analytical device that consists of a biorecognition element which can specifically interact with an analyte and produce physical, chemical or electrical signals.

Conducting polymers (CPs) or, more precisely, intrinsically conducting polymers are organic polymers that conduct electricity, having either metallic conductivity or semiconductor properties. CPs can exhibit mixed conduction which is the ability to conduct both ionic and electronic current.

Conjugated polymers contain alternating single and double bonds on the polymer backbone allowing stabilization of the structure of the molecule upon addition or subtraction of a charge, through redox activation, conferring on it electronically conducting behavior. These polymers when subjected to redox activation generate polyionic structures in which charge compensation takes place, in a similar manner to the doping in inorganic semiconductors.

Direct electron transfer (DET) involves direct electron transfer between the redox active center of the catalytic biorecognition element (e.g. enzyme) and the electrode in the absence of an electron transfer mediator. A Transducer is a device that converts the biological signal that is generated to a readable signal: electrical, optical or mechanical.

Electrochemical enzyme-based sensing relies on coupling a redox reaction catalyzed by an enzyme with an electrochemical transducer using techniques such as amperometry, potentiometry, and voltammetry. Mediated electron transfer (MET) occurs with the help of small molecules that shuttle electrons between the biorecognition element and the electrode.

Metabolites are the intermediates and products of metabolism.

Multiplexing involves the incorporation of multiple sensing capabilities in terms of analyte diversity

Organic electrochemical transistors (OECTs) are electrolyte-gated organic thin film transistors (EGTs) where, unlike the conventional EGTs, the doping level modulation occurs in the bulk of the CP film resulting in especially high currents for very small gate fluctuations (sub Volts), a property also known as transconductance. When coupled with biorecognition elements, this inherent amplification results in highly sensitive transduction of a biological event i.e., the enzymatic detection of metabolites.

Permselective membranes are ion-exchange materials that allow ions of one polarity to enter and pass through.

Poly(3,4-ethylenedioxythiophene)-poly(styrenesulfonate) (PEDOT:PSS) is an intrinsically doped p-type semiconductor and is the champion material used to date in OECTs; transistors shown to exhibit the highest transconductance values among their electrolyte-gated counterparts. 


\section{Biosensors as Analytical Tools in Point-of-care Diagnostics}

Healthcare spending represents today $18 \%$ of the GDP in the US (https://www.cms.gov), underlying the need for new, more reliable low-cost and easy-to-use diagnostic tools. Parallel developments in biomarker research and diagnostic technologies, are anticipated to shift the existing "one-size-fits-all

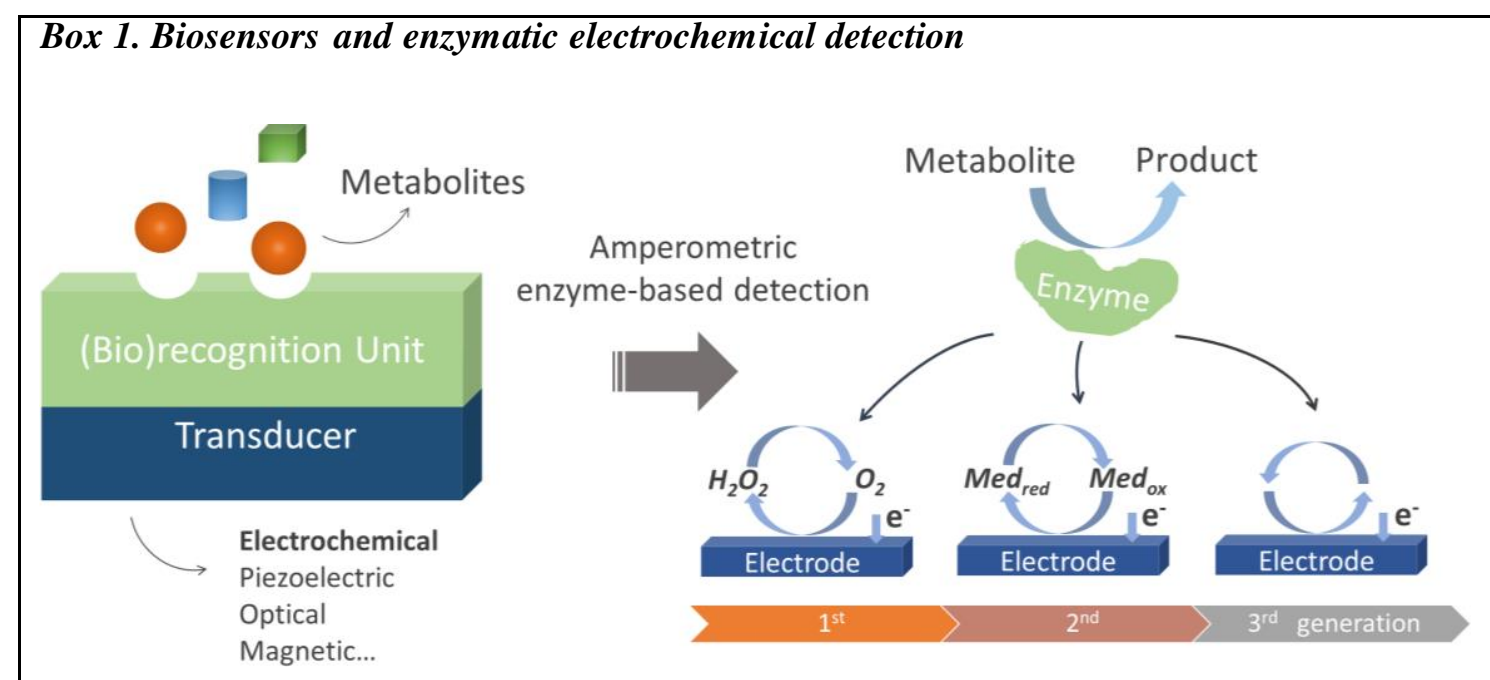

Caption: General outline of a biosensor and the evolution of the different generation of amperometric enzyme biosensors based on the electron transfer between the enzyme and the electrode surface. What is a biosensor? The biosensor, first introduced by Leland C. Clark Jr. and co-workers in 1962 [1], is defined as an analytical device incorporating a biorecognition element within or associated with physiochemical transducers (classified as optical, electrochemical or mechanical [2-4]).

What does a biosensor do? It generates a digital electronic signal based on the concentration of specific chemicals or end products of a given biochemical reaction in an analytical standard [5].

What is a biosensor used for? Applications ranging from medical diagnostics to drug discovery, food safety, process control and environmental monitoring, to defense and security applications [6].

Electrochemical techniques allow for fabrication of portable, miniaturized, low-cost, often label-free and simple-to-operate analytical devices that interface with biological components [7-9]. The working mechanism relies on selective recognition of a target analyte by a biomolecule or bio-macromolecular complex, which in turn produces a measurable electrochemical signal. Although a variety of biorecognition elements can be employed, electrochemical detection techniques rely predominantly on enzymes. Enzyme-based electrochemical biosensors are typically preferred for metabolite monitoring due to the highly selective catalytic activity and fast acting properties of the enzymes at the electrode interface [10-12].

approach" in the global healthcare system to a more proactive and personalized model that also

facilitates data-processing and portability ("connected body") [13]. Modern biosensors, (see Glossary)

with a market size of a US\$ 13 billion annual turnover, have rapidly become an invaluable tool in the

healthcare industry [14]. Indeed, glucose biosensors (accounting for $85 \%$ of the total biosensor market)

have significantly improved the quality of everyday life of diabetic patients, with considerable socio- 
economic impact beyond the single patient outcome [15]. As with most state-of-the-art healthcare devices, biosensors rely heavily on in vitro measurements from bodily fluids (i.e., blood, saliva, tears etc). The increasing adoption of wearable technologies in healthcare as well as the concept of closedloop continuous real-time monitoring systems that can provide real time information and therapeutic strategies on demand at the point-of-care, is nonetheless anticipated to shift this trend towards on body or/and in vivo technologies. However, technical hurdles and limitations still impede the practical applicability and commercialization of those futuristic concepts, including continuous and multiplexed monitoring, non-invasiveness, portability, long-term stability, ease in device integration and compatibility with flexible substrates [14- 17]. Novel technologies to overcome those limitations are urgently needed and as most technologies are limited by the materials used, materials research and development is anticipated to be the key driver in next generation biosensors.

In this review, recent advances in enzyme-based electrochemical detection of human metabolites in real samples using organic electronic materials are described. Electrochemical methods offer a number of advantages over optical methods, mostly due to the rapid and label-free detection schemes. Moreover, when coupled with enzymes, their high selectivity and excellent catalytic activity renders electrochemical enzyme- based sensing one of the most promising methods for the detection of a wide repertoire of metabolites [Box 1]. In such approaches, the selectivity and sensitivity of the biosensors can be sought by establishing an exclusive and intimate interface between the transducer and the enzyme thus reducing interference as well as improving the efficiency of the electron transfer (ET) involved in the redox reactions [18]. Hence, the concept of "functional" electronic materials that can simultaneously provide an ideal immobilization matrix and an efficient signal transducer has fundamentally changed the understanding of how to develop efficient biosensors [19]. We thus place herein a particular emphasis on the promising class of polymer-based electronics and more specifically electrically conducting polymers (CPs). In the cases where such examples are still scarce, we showcase alternatives with carbon-based nanostructures, particularly where their operation in real world applications pushes the state of the art thanks to their materials properties. We highlight the key properties that render organic electronic materials ideal transducers both for metabolite monitoring, 
including better electrochemical communication at the bio-electronic interface, faster electron transfer, lower signal-to-noise ratios, etc., and for integrating these systems in real-world applications. Finally, we discuss the niche for organic electronic metabolite sensors for state-of-the-art point-of-care diagnostics (POC), as categorized into three groups; in vitro, wearable, and implantable biosensors.

\section{Organic Electronics at the Interface with Biology: state of the art in metabolite sensing}

\section{Box 2. Organic Electronic Materials}

Organic electronic materials, including conjugated polymers, small molecules and carbon based nanomaterials, exhibit multiple advantages over their inorganic equivalents, such as ease in processing, compatibility with flexible substrates, and low-cost and environmentally-friendly manufacturing [20-23]. Organic electronics are not only ideal substrates for chemical modification and bioreceptor immobilization but also excellent electronic signal transducers because of their literal flexibility and tunability to adapt to challenging performance requirements in biological milieus [2427], as well as their intrinsic unique properties including high surface-to-volume ratio (owing to their nanometer scale molecular structures), high electrical conductivity, chemical stability, and excellent mechanical properties $[28,29]$.

Conducting polymers constitute an especially promising class of electronic materials. The doping or de-doping of such conjugated polymers can be reversible and triggered by chemical, electrical or biological events [30, 31] showing great potential for applications such as biosensing [32, 33]. Polypyrrole (PPy), polyaniline (PANI), and poly (3,4-ethylenedioxythiophene) (PEDOT), are the predominantly studied CPs in biological applications due to their biocompatibility and ease of processing.

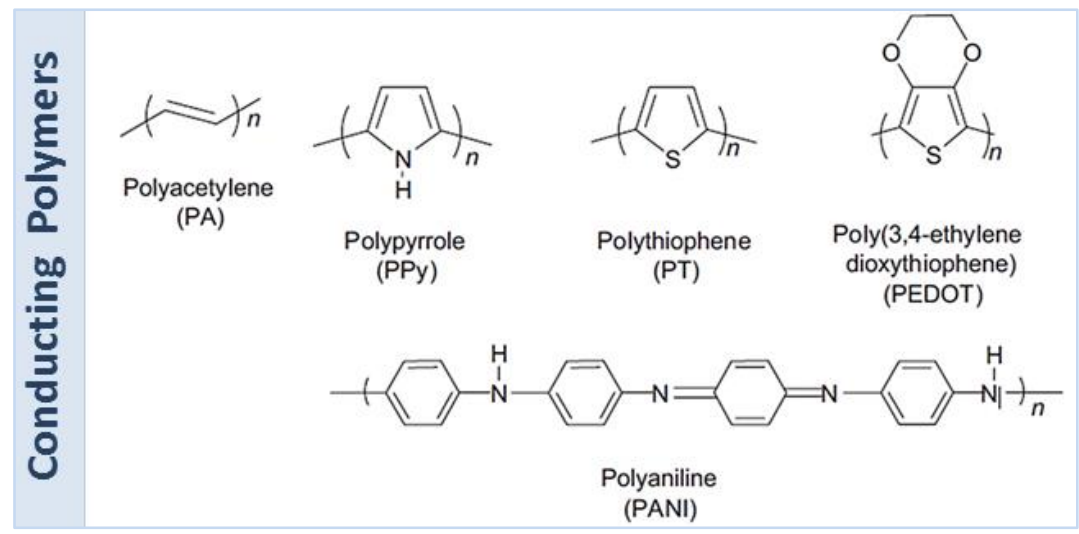

Caption: Chemical structures of the most commonly studied conducting polymers

The ability to operate in harsh biological environments, enhanced sensitivity and selectivity, and demonstrated potential for low cost production, are just some of the reasons why organic electronic materials are poised to revolutionize the biosensing arena (see Box 2).

\subsection{Conducting polymers as efficient electron relays}


Efficient electrical communication between the biorecognition element (e.g. an enzyme) and the electrode is rather challenging when designing enzymatic biosensors. In most cases, the distance between the active site of the enzyme and the electrode surface is too long for direct electron transfer (DET), due to the enzyme's protective shield. Since electron transfer (ET) via a tunneling mechanism is rarely encountered in traditional electrodes, establishing electron relays that allow for fast ET, avoiding free-diffusing redox species between the electrode and the enzyme, is crucial [34]. In this context, organic electronic materials represent very promising candidates for molecular wiring due to their polymeric nature and conducting behavior [35]. Their ability to be electrochemically polymerized in the presence of biologically active molecules, also allows for an intimate and spatially controlled enzyme localization inside their matrix, substantially improving enzyme-electrode coupling $[35,36]$.

Doped PPy was the first conducting polymer shown to provide an electron relay between the surface of the electrode and the active site of an enzyme, significantly enhancing the biosensor's analytical characteristics $[37,38]$. However, due to poor electrochemical stability (potentially affecting long-term functionality) [39], efforts shifted to other materials, such as PEDOT, a polythiophene derivative which emerged as a more stable candidate due to its low bandgap and high electrochemical stability in the oxidized state [40]. The first example of a PEDOT-based glucose sensor with potential for long-term measurements was presented by Kros and colleagues [41]. They physically incorporated a positively charged polymer in the conducting matrix of the biosensor, allowing for more efficient ET due to the increased electrostatic interaction of the positively charged entrapped polymer with the negatively charged enzyme (Figure 1a). In a more direct approach, Thompson and colleagues compared chemical vs electrochemical incorporation of the enzyme glucose oxidase (GOx) directly into PEDOT during polymerization, concluding that the chemical polymerization resulted in more efficient enzyme entrapment and thus DET [42].

An alternative strategy to improve the electron relay in CPs post-synthesis involves intermixing with redox hydrogels, which have been shown to exhibit fast substrate and counter-ion diffusion properties with high flexibility and fast electron transfer rates. The non-conducting nature of such hydrogels impedes their efficient and spatially localized immobilization on the active electrode surface, 
so combining them with CPs can overcome this issue resulting in an ideal electron-transfer pathway. PEDOT:PSS was used to enhance the poor performance of a mediator based biosensor by its incorporation in nanocomposite enzyme electrodes, resulting in improved electron hopping in terms of the electron diffusion coefficient and charge transfer resistance (Figure 1b) [43]. Going one step further, the Bao group developed intrinsically conducting nanostructured PANI-redox hydrogels. The high surface area and interconnected hydrogels not only resulted in excellent electronic conductivity and electrochemical properties but also served as an efficient catalytic substrate for the enzymatic determination of glucose, yielding excellent analytical biosensor properties [44]. In another approach, a CP-based glucose-permeable redox hydrogel was formed by cross-linking polymer acid-templated PANI along with GOx, leading to the electrical wiring of the enzyme and allowing the electrocatalytic oxidation of glucose at low oxidation potentials (Figure 1c). [45] More recently, CP hydrogels with high permeability to enzymes were used to fabricate metabolite biosensors with excellent sensing performance without the need for a mediator (Figure 1d). [46]

\subsection{Conducting polymers as permselective membranes for improved selectivity}

Another striking feature of organic electronic materials is their potential to be used as permselective membranes to eliminate interference induced by electroactive compounds, which is particularly important for real-world applications due to the increased complexity of the biological milieu. As such, overoxidized polypyrrole (OPPy) has been employed to develop interference-free biosensors as a cation exchange film that can repel common interferents found in complex media and also limit protein binding due to the oxygen-containing groups introduced onto its surface. Additionally,

controlled deposition of OPPy via electropolymerisation is a versatile approach for creating permselective membranes [47]. PANI-polyisoprene films have been also used as immobilization matrices for GOx, and the resulting composite film was found to exhibit high permselectivity, allowing selective $\mathrm{H}_{2} \mathrm{O}_{2}$ detection over common electroactive interferents [48].

\subsection{Nanostructured organic electronic materials for improved sensitivity}


Arguably, nanostructured sensing elements provide higher sensitivity due to increased surfaceto-volume ratios. Due to the versatility in processing organic electronic materials, nanoscale structures can be easily produced to improve both the enzyme immobilization efficiency and the sensitivity and stability of the sensor. Yang and coworkers recently developed PEDOT nanofibers carrying GOx on top of a Pt electrode, resulting in increased entrapment of the enzyme owing to its nanoscale matrix and a reduction in the electrode impedance, achieving glucose detection at relatively low polarization potentials [49].

\subsection{Conducting Polymer-based devices}

Organic electronics have been used for enhancing biosensors thanks to their distinct materials properties, but they can themselves constitute the active material of the transducer, resulting in highly integrated devices. Forzani and colleagues developed a glucose sensing device comprised of interdigitated nanoelectrodes, bridged by PANI/GOx nanojunctions. The transduction mechanism relied on glucose oxidation inducing changes in the polymer redox state. Due to the small size of the nanojunction sensor, the enzyme was naturally regenerated without the need for redox mediators, allowing for very fast responses and minimal oxygen consumption, which is a prerequisite for future in vivo applications [50]. Mixed conduction in CPs is a key property that has enabled several new modes of operation and devices [51], including the organic electrochemical transistor (OECT), a rising star in terms of biosensing applications [52, 53]. After the first work on glucose sensing with OECTs in 1998, based on the CP PANI, using tetrathiafulvalenium as an electron transfer mediator [54], many have followed, mainly aiming at detecting $\mathrm{H}_{2} \mathrm{O}_{2}$ catalyzed oxidation at a Pt gate. [55] Integrating an ionic liquid as an electrolyte [56] or incorporating carbon-based nanomaterials (such as graphene oxide) at the gate and channel electrode [57] represent interesting attempts to improve the stability and sensitivity of such devices (Figure 2 a,b). OECT-based sensors have also been screen-printed and functionalised using a chitosan hydrogel matrix, incorporating a ferrocene electron transfer mediator along with the corresponding oxidase enzyme for the detection of glucose and lactate on artificial sweat samples (Figure 2c). [58] Epinephrine, a key neurotransmitter, has been also detected using solution-processed 
OECTs with remarkable sensitivities (sub-nM) owing to a Nafion anti-interference layer and carbonbased nanomaterials co-immobilized on the gate electrodes (Figure 2d) [59].

\section{Highly integrated Organic Electronic Devices for Point of Care Metabolite Sensing}

\subsection{In vitro metabolite biosensors}

\section{Metabolite sensing from bodily fluids/ gases}

Blood is the most commonly used bodily fluid for metabolite level monitoring. However, due to the abundance of electroactive species, electrochemical detection methods become somewhat challenging, and the commonly observed biofouling of the sensing electrodes poses further restrictions. [60] CPs bearing appropriate surface modifications (i.e., incorporation of electron mediators, permselective membranes, etc.) can offer valuable tools towards novel, more accurate diagnostic devices. An antibodymediated amperometric system was designed by Wei and colleagues to avoid the interfering signals often encountered in complex matrices such as whole blood when using enzymatic-mediated amperometric detection. PPy matrix served for the immobilization of the capture antibody, on top of a 16-array gold electrochemical sensor, which could therefore detect creatinine rapidly and accurately in whole blood, resulting in a POC assay for allograft dysfunction (Figure 3a). [61]

Liao and coworkers recently developed a flexible OECT platform based on PEDOT:PSS to selectively detect urea and glucose in saliva samples. [62] To eliminate electrochemical interference in saliva, thus enhancing sensitivity and selectivity, the gate electrodes were modified with oppositely charged bilayer polymeric films for both anionic and cationic charge exclusion of interferents. Moving towards multiplexing, a PEDOT:PSS based OECT biosensing platform integrated with microfluidics was developed for simultaneously screening glucose, lactate and cholesterol in human saliva samples. The interference issues were resolved by operating the device at a bias far below the oxidation potential of the electroactive species present in biofluids, by using ferrocene derivatives as a mediator. The final device was tested with human volunteers before and after exercise to show relative variations in their metabolite profiles under stimuli (Figure 3b). [63] In a similar approach, simultaneous sensing of lactate and glucose was demonstrated by integrating two OECT-based devices, each with a separate 
microfluidic channel. They generated a prototype portable glucose sensor by linking a smartphone with the device through Bluetooth connection, highlighting the ease of integration of such devices for POC systems. [64]

Electronic devices (e-noses) have emerged as excellent candidates for detecting breath volatile biomarkers compared with conventional methods (i.e., breathalyzers) used to date. Pavlou and colleagues were the first to detect the pathogen Mycobacterium tuberculosis both in vitro and in situ using a 14 gas-sensor array. This array consisted of a set of specifically tailored CPs that physically interact with volatile compounds produced by the in vitro cultures or the sputum samples, resulting in a change in electrical resistance. [65] In another study, chemically polymerized PPy films on chips were used to capture absorbing and desorbing breath volatiles for subsequent chemical analysis both from environmental air samples and directly from exhaled human breath, paving the way for lab-on-a-chipbased environmental and health monitoring systems. [66] Bihar and colleagues recently developed a printed disposable breath analyser based on an all-PEDOT:PSS printed OECT for detecting blood alcohol levels from breath. The sensing platform, tested on human volunteers, showed remarkable sensitivity in detecting the consumption of even one glass of wine. (Figure 3c). [67]

\section{Metabolite sensing from whole cells}

Detecting cellular metabolites under different stimuli or environmental conditions can give useful insights for drug discovery and toxicology, particularly with the advent of organ-on-chip technologies. Larsen and coworkers used PEDOT:tosylate microelectrodes as an all polymer electrochemical chip for simple detection of potassium-induced transmitter release from neuron-like cells, demonstrating the potential of the method for drug screening applications. [68] To improve the electrocatalytic activity of the sensing electrode, the PEDOT:PSS gate may also be decorated with electrodeposited Pt nanoparticles (NPs). [69] Due to the high surface area of the NPs and the enzymes' specificity, the authors achieved very sensitive determination of the critical metabolites glucose and lactate from live cells. Lactate production in tumor cell cultures derived from real patients was also measured using an OECT circuit. To circumvent the issue of interference in such samples, the proposed reference based 
sensor circuit design allowed for inherent background subtraction. Lactate production could be measured from a few cells underlying the sensitivity of the device over a highly complex milieu, thus illustrating its potential for its use in in vivo applications for cancer diagnostics (Figure 3d). [70] In a more recent study, Curto and colleagues demonstrated a multiparametric on-chip platform integrated with microfluidics for cell cultures, using among other in-line methods the OECT-based detection of glucose produced by the cells as a measure to validate their improved differentiation under stimuli conditions. [71]

\subsection{Wearable Metabolite Biosensors}

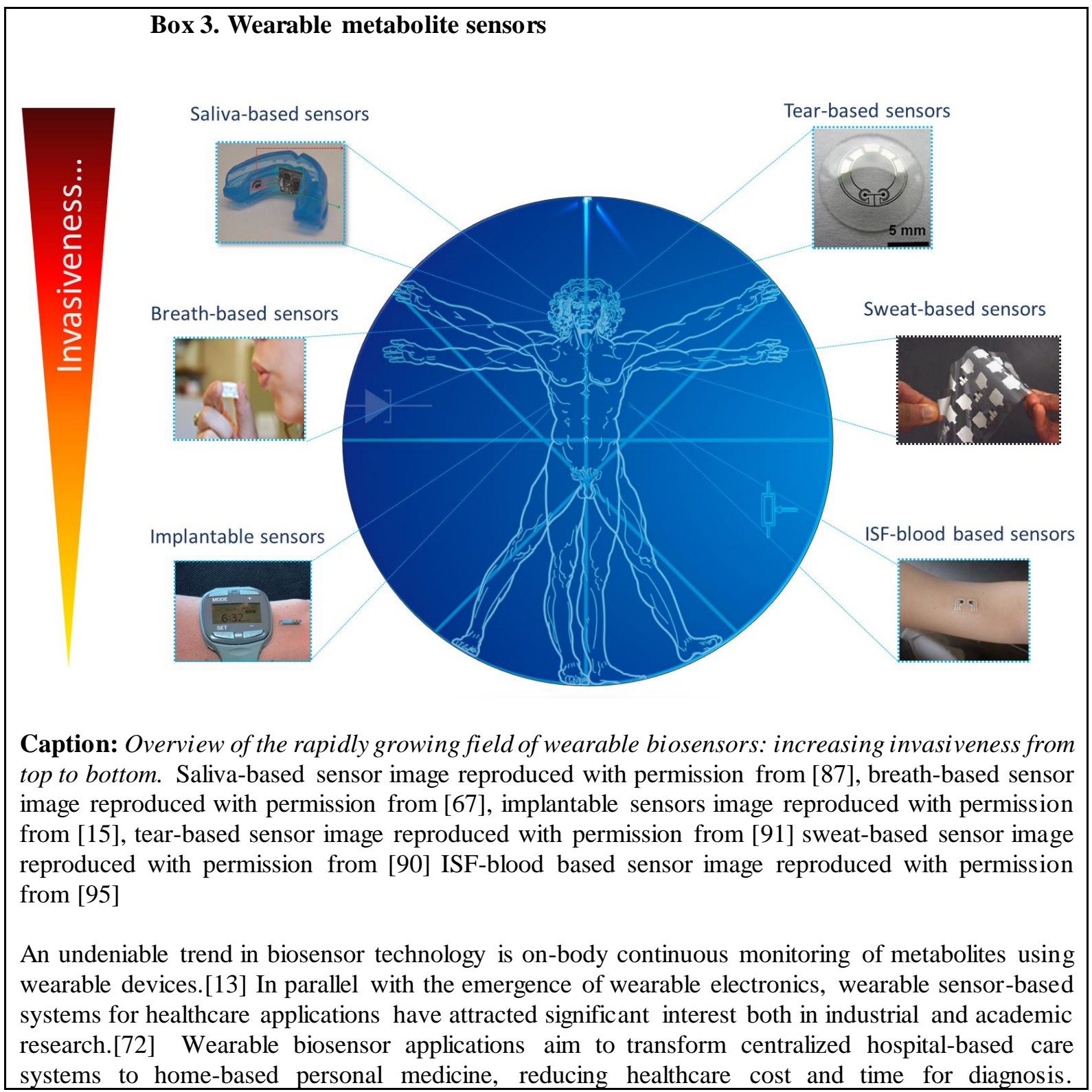


Electrochemical transducers offer many advantages for wearable sensors for physiological monitoring, and can be easily integrated onto textile materials or directly on the skin.

The move towards biosensor technology for on-body continuous metabolite monitoring and the advancements in wearable electronics [13] has inevitably led to wearable sensors for healthcare applications (see Box 3) [6, 72]. Although organic electronic materials have been successfully implemented into wearable sensors for electrophysiological measurements $[73,74]$, there are currently few examples of metabolite sensors using this technology [75]. As we expect this area to advance rapidly, we present here some examples of current technologies used for metabolite sensing in wearable formats and illustrate progress towards this goal using organic electronic materials. Sweat-based wearable sensor systems, although mostly focused on a small number of physical or electrophysiological parameters, can yield significant information about a patient's health status based on levels of some critical metabolites [76]. Wearable biosensors can be either textile/plastic based or epidermal (tattoo)based systems [77]. Epidermal biosensors provide better contact with skin but usually exhibit shorter lifetimes compared to textile-based systems. Such biosensors were first developed in 2009 by Kim and colleagues for continuous monitoring of physical parameters [78] and shortly after, Jia and colleagues combined this approach with biorecognition elements towards the first printed tattoo-based biosensor [79]. A screen-printed electrode on temporary tattoo paper was developed with carbon and $\mathrm{Ag} / \mathrm{AgCl}$ serving as the working and reference electrodes, respectively. The working electrode was also modified with CNTs carrying a mediator together with lactate oxidase (LOx) for continuously monitoring lactate in sweat during exercise [79]. The same group later introduced a fully integrated sensor (comprising a sensory part, a microcontroller, a wireless communication system and an acquisition system) in an epidermal patch. The sensory part was composed of a screen-printed three-electrode-based amperometric sensor and two carbon-based electrocardiogram electrodes for the simultaneous measurement of lactate and electrophysiological parameters (Figure 4a). [80]

CPs are especially advantageous for wearable sensor technology due to their compatibility with fabrication on flexible substrates. [56] In a very interesting approach, Pal and colleagues developed 
PEDOT:PSS electrodes on flexible fully biodegradable silk protein fibroin supports using a simple photolithographic process and an aqueous ink composed of the $\mathrm{CP}$ and carrier proteins. (Figure $4 \mathbf{b}$ ). [81] The silk provided excellent mechanical and optical properties along with biocompatibility and controllable degradation. In a similar approach by the same group, silk proteins including fibroin and sericin were modified with photoreactive methacrylate groups to be used as substrate inks for the waterdispersable PEDOT:PSS that was micropatterned to develop biodegradable bioelectrode, for glucose sensing in vitro [82]. This approach opens up a new direction for fabricating an entirely organic, freestanding device with controllable biodegradability including scalability and processability, leading to applications in wearable or implantable bioelectronics with "wear/implant and forget" functionality [83].

Complementing recent progress on in vitro salivary diagnostics, wearable salivary sensing has also been reported, dating back to the 1960 s, where $\mathrm{pH}$ and other important electrolytes were measured on a partial denture. $[84,85]$ However, the focus now is on the development of wearable sensors for the early diagnosis of important metabolic diseases. $[13,86]$ An early example is a non-invasive mouthguard biosensor for continuously monitoring uric acid, the end product of purine metabolism in the human body, developed by Wang and coworkers [87]. This study developed a screen-printed amperometric enzyme-based biosensor together with an integrated wireless transmitter. Carbon electrode-containing mediators were printed as transducing elements on a plastic substrate, and crosslinked uricase enzyme was electropolymerized to serve as the biorecognition element, for sweat sensors. A wireless amperometric circuit coupled with a Bluetooth low-energy communication system was integrated on the mouthguard biosensor as shown in Figure 4c. The advantages of this mouthguard sensor over indwelling types of devices are wearability, ease of operation and renewability.

The very first successful prototype of electronic contact lenses was developed by Parviz and colleagues in 2009, showing the first in vivo test of a wirelessly powered contact lens display placed on a live, anesthetized rabbit's eye. [88] Later, they developed the first metabolite sensor in human tear fluid using the same contact lens approach by integrating a three-electrode amperometric sensor system consisting of a Pt working electrode with immobilized $\mathrm{GOx}$, and an external $\mathrm{Ag} / \mathrm{AgCl}$ reference 
electrode on plastic films in an electrochemical cell, again detecting $\mathrm{H}_{2} \mathrm{O}_{2}$ catalysis. [89, 90] A lactate biosensor was developed in the same manner, replacing GOx with LOx [91]. Mitsubayashi and colleagues developed an interesting strategy to produce enzyme-based glucose biosensors by integrating a polydimethyl siloxane (PDMS) membrane onto a contact lens. The counter/reference electrodes were fabricated by successively sputtering $\mathrm{Ag}$ and Pt metal onto a $70 \mu \mathrm{m}$ thick PDMS substrate (Figure 5ab). [92] Flexible electrodes were then bonded onto the surface of the contact lens using PDMS, and GOx was immobilized on the sensing region of the electrode. This integrated biosensor was successfully tested using a rabbit model. [92] Progress is moving towards integrating organic electronic materials into metabolite sensors from tears; a recent study by Lee and coworkers illustrated the potential for integrating graphene into contact lenses [93], while Tehrani and coworkers are working towards rapid detection of glucose on CuNP decorated graphene electrodes. [94]

Even though there has been no commercial success so far in non-invasive wearable metabolite sensing using saliva, tears or sweat, limited commercial success has been achieved with interstitial fluid (ISF) based biosensors. One of the best-known examples is the GlucoWatch Biographer ${ }^{\mathrm{TM}}$ (Cygnus, Inc.). Having initially attracted considerable attention in the early 2000 s, it was quickly withdrawn due to significant inaccuracies, sampling errors, skin irritation problems and frequent calibration needs [15]. Recently, Abbott launched the FreeStyeLibre (www.freestylelibre.com) as a new wearable continuous glucose monitoring device, showing more promise in terms of comfort and user interface; the sensor can be used for 14 days and does not require any finger-stick calibration, while its small sensory part (0.2 inches long) directly connects to a plastic patch that can be easily placed on the upper arm. The glucose concentration is transferred in real-time to an external reader wirelessly via a near-infrared identification tag. This recent progress on ISF based biosensors further expands the wearable biosensor market and is anticipated in the near future to extend to other metabolites beyond glucose, offering multiplexing capabilities toward personalized diagnostics.

\subsection{Implantable Biosensors}


The concept of implantable metabolite sensors was first proposed by Leyland C. Clark in his seminal paper in 1962 [1] but was put into practice by Shichiri and colleagues in the early 1980s [95]. There have been many different research efforts since then, mostly focusing on developing continuous glucose monitoring systems [96]. One prominent study described an implantable $100 \mathrm{~mm}$ flexible biochip for intravenous glucose sensing in rat blood. The fabricated microelectrode was modified with a carbon-based composite consisting of flexible carbon fibres, neutral red as a mediator and GOx as an enzyme layer. The device was inserted in the thoracic region of a living rat for glucose measurements, simulating diabetic conditions by injecting glucose intravenously (Figure 5c) [97].

Despite limited progress in academic studies, considerable success has been achieved in industrial research, and the implantable biosensor market has begun to expand in the last decade. In 2005, Medtronic (USA) launched the first implantable continuous glucose monitoring device for personal use $[6,15]$. The self-implanted sensor was based on the amperometric determination of $\mathrm{H}_{2} \mathrm{O}_{2}$ produced by the oxidation of glucose in the presence of glucose oxidase. Despite the commercial and medical success, there are still restrictions that to some extent limit the use of such devices. For instance, the FDA still stipulates that the finger-stick blood test must be performed in parallel when using these self-implantable biosensors [15]. However, this limitation was very recently overcome by the same company, with the world's first FDA approved hybrid closed loop (HCL) insulin delivery system, the MiniMed 679G. This new device provides automatic adjustment of basal insulin delivery without requiring a finger-stick test, opening up new avenues in the area of implantable biosensors for continuous monitoring of glucose (www.medtronicdiabetes.com). While it is impossible to include all successful devices in this review, other reviews describe more about the historical evolution and development of commercialised implantable biosensors [6, 15-19, 98].

\section{Conclusions}

Despite the significant progress in biosensor development, successfully commercialized devices are still scarce, with glucose sensors a notable exception. The dual market demands of high analytical performance and cheap, easy-to-use, long-lasting devices often render their clinical implementation beyond the reach of current technologies. Organic electronics is a field of research that has rapidly come 
to the fore for biological applications, despite its beginnings in large-area, flexible applications embodied by devices such as the organic light emitting diode or organic photovoltaics. One of the factors credited for the success of organic electronics in biosensing applications is the literal flexibility and tunability of these materials to adapt to challenging performance requirements in biological milieus. Organic electronics may therefore represent the ideal technology to meet the demands of the biosensor market (see Outstanding Questions). Indeed, in cases of bioelectrocatalytic detection methods—such as those described in this review-we show examples where organic, often polymeric structure can allow for molecular wiring with the enzyme's active site and thus achieve high sensitivities without the need for artificial electron mediators. Bearing in mind commonly raised issues in terms of stability and lifetime of the enzymes, future trends in this dynamic, fast-moving field of organic electronic biocatalytic sensors are anticipated to include the development of biomimetic architectures (i.e., molecular imprinted structures), harnessing the versatility in synthesis of such electronic materials. Transducer and biorecognition elements can be envisioned as a single active component, combining electronic functionalities and the best features of the biological reagent in a more stable matrix and opening up new exciting directions in biosensor technology in both fundamental and practical aspects.

\section{Acknowledgements}

A.M.P and R.O gratefully acknowledge the support by the Marie Curie ITN project OrgBio No. 607896. O.P. gratefully acknowledges support from the Knut and Alice Wallenberg Foundation (KAW 2014.0387) for postdoctoral research at Stanford University. 


\section{References}

1. Clark, L.C., Jr. and Lyons, C. (1962) Electrode systems for continuous monitoring in cardiovascular surgery. Annals of the New York Academy of Sciences 102, 29-45

2. Shukla, S.K., et al. (2014) Self-Reporting Micellar Polymer Nanostructures for Optical Urea Biosensing. Industrial \& Engineering Chemistry Research 53, 8509-8514

3. Parlak, O., et al. (2017) Structuring Au nanoparticles on two-dimensional MoS2 nanosheets for electrochemical glucose biosensors. Biosensors \& Bioelectronics 89, 545-550

4. Parlak, O., et al. (2014) Two-Dimensional Gold-Tungsten Disulphide Bio-Interface for HighThroughput Electrocatalytic Nano-Bioreactors. Advanced Materials Interfaces 1

5. Scheller, F. and Schubert, F. (1991) Biosensors. Elsevier Science

6. Turner, A.P.F. (2013) Biosensors: sense and sensibility. Chemical Society Reviews 42, 3184-3196

7. Bard, A.J. and Faulkner, L.R. (2000) Electrochemical Methods: Fundamentals and Applications. Wiley

8. Jay, C.R.G.W., et al. (2013) Electrochemistry. Royal Society of Chemistry

9. Kleijn, S.E.F., et al. (2014) Electrochemistry of Nanoparticles. Angewandte Chemie-International Edition 53, 3558-3586

10. Parlak, O., et al. (2013) Template-directed hierarchical self-assembly of graphene based hybrid structure for electrochemical biosensing. Biosensors \& Bioelectronics 49, 53-62

11. Osikoya, A., et al. (2016) Acetylene Sourced CVD synthesized catalytically active graphene for electrochemical biosensing. Biosensors and Bioelectronics

12. Kimmel, D.W., et al. (2012) Electrochemical Sensors and Biosensors. Analytical Chemistry 84, 685-707

13. Hamburg, M. A., and Collins F. S., (2010) The Path to Personalized Medicine. The New England Journal of Medicine 363, 301-304

14. Sekretaryova, A.N., et al. (2016) Bioelectrocatalytic systems for health applications. Biotechnology Advances 34, 177-197

15. Gifford, R. (2013) Continuous Glucose Monitoring: 40 Years, What Weve Learned and What's Next. Chemphyschem 14, 2032-2044 
16. Newman, J.D. and Turner, A.P.F. (2005) Home blood glucose biosensors: a commercial perspective. Biosensors \& Bioelectronics 20, 2435-2453

17. Wilson, G.S. and Gifford, R. (2005) Biosensors for real-time in vivo measurements. Biosensors \& Bioelectronics 20, 2388-2403

18. Owens, R.M. and Malliaras, G.G. (2010) Organic Electronics at the Interface wtith Biology. Mrs Bulletin 35, 449-456

19. Rivnay, J., et al. (2014) The Rise of Organic Bioelectronics. Chemistry of Materials 26, 679-685

20. Mathieson, K., et al. (2012) Photovoltaic Retinal Prosthesis with High Pixel Density. Nat Photonics 6, 391-397

21. Someya, T., et al. (2016) The rise of plastic bioelectronics. Nature 540, 379-385

22. Lanzani, G. (2014) Materials for bioelectronics: organic electronics meets biology. Nat Mater 13, 775-776

23. Pitsalidis, C., et al. (2016) High mobility transistors based on electrospray-printed smallmolecule/polymer semiconducting blends. Journal of Materials Chemistry C 4, 3499-3507

24. Bettinger, C.J. and Bao, Z. (2010) Organic thin-film transistors fabricated on resorbable biomaterial substrates. Adv Mater 22, 651-655

25. Takamatsu, S., et al. (2016) Wearable Keyboard Using Conducting Polymer Electrodes on Textiles. Adv Mater 28, 4485-4488

26. Palazzo, G., et al. (2015) Detection Beyond Debye's Length with an Electrolyte-Gated Organic Field-Effect Transistor. Advanced Materials 27, 911-916

27. Kim, S.H., et al. (2013) Electrolyte-Gated Transistors for Organic and Printed Electronics. Advanced Materials 25, 1822-1846

28. Adhikari, B.R., et al. (2015) Carbon Nanomaterials Based Electrochemical Sensors/Biosensors for the Sensitive Detection of Pharmaceutical and Biological Compounds. Sensors (Basel) 15, 22490-22508

29. Yang, W., et al. (2010) Carbon Nanomaterials in Biosensors: Should You Use Nanotubes or Graphene? Angewandte Chemie International Edition 49, 2114-2138

30. Chiang, C.K., et al. (1977) Electrical Conductivity in Doped Polyacetylene. Physical Review Letters 39, 1098-1101

31. Bredas, J.L. and Street, G.B. (1985) Polarons, bipolarons, and solitons in conducting polymers. Accounts of Chemical Research 18, 309-315 
32. Crispin, X., et al. (2006) The Origin of the High Conductivity of Poly(3,4ethylenedioxythiophene)-Poly(styrenesulfonate) (PEDOT-PSS) Plastic Electrodes. Chemistry of Materials 18, 4354-4360

33. Malhotra, B.D., et al. (2006) Prospects of conducting polymers in biosensors. Anal Chim Acta 578, 59-74

34. Sekretaryova, A.N., et al. (2014) Unsubstituted phenothiazine as a superior water-insoluble mediator for oxidases. Biosensors \& Bioelectronics 53, 275-282

35. Vallejo-Giraldo, C., et al. (2014) Biofunctionalisation of electrically conducting polymers. Drug Discov Today 19, 88-94

36. Schuhmann, W. (2002) Amperometric enzyme biosensors based on optimised electron-transfer pathways and non-manual immobilisation procedures. J Biotechnol 82, 425-441

37. Schuhmann, W., et al. (2000) Electron-transfer pathways between redox enzymes and electrode surfaces: reagentless biosensors based on thiol-monolayer-bound and polypyrroleentrapped enzymes. Faraday Discuss, 245-255; discussion 257-268

38. Swann, M.J., et al. (1997) The role of polypyrrole as charge transfer mediator and immobilization matrix for $d$-fructose dehydrogenase in a fructose sensor. Biosensors and Bioelectronics 12, 1169-1182

39. Pud, A.A. (1994) Stability and degradation of conducting polymers in electrochemical systems. Synthetic Metals 66, 1-18

40. Kros, A., et al. (2005) Poly(pyrrole) versus poly(3,4-ethylenedioxythiophene): implications for biosensor applications. Sensors and Actuators B: Chemical 106, 289-295

41. Kros, A., et al. (2001) Poly(3,4-ethylenedioxythiophene)-Based Glucose Biosensors. Advanced Materials 13, 1555-1557

42. Thompson, B.C., et al. (2010) Conducting Polymer Enzyme Alloys: Electromaterials Exhibiting Direct Electron Transfer. Macromolecular Rapid Communications 31, 1293-1297

43. Wang, J.-Y., et al. (2013) Synthesis of Redox Polymer Nanobeads and Nanocomposites for Glucose Biosensors. ACS Applied Materials \& Interfaces 5, 7852-7861

44. Pan, L., et al. (2012) Hierarchical nanostructured conducting polymer hydrogel with high electrochemical activity. Proceedings of the National Academy of Sciences 109, 9287-9292

45. Mano, N., et al. (2007) An Electron-Conducting Cross-Linked Polyaniline-Based Redox Hydrogel, Formed in One Step at pH 7.2, Wires Glucose Oxidase. Journal of the American Chemical Society $129,7006-7007$

46. Li, L., et al. (2015) A nanostructured conductive hydrogels-based biosensor platform for human metabolite detection. Nano Lett 15, 1146-1151 
47. Centonze, D., et al. (1992) Interference-free glucose sensor based on glucose-oxidase immobilized in an overoxidized non-conducting polypyrrole film. Fresenius' Journal of Analytical Chemistry 342, 729-733

48. Xue, H., et al. (2001) Polyaniline-polyisoprene composite film based glucose biosensor with high permselectivity. Synthetic Metals 124, 345-349

49. Yang, G., et al. (2014) High-Performance Conducting Polymer Nanofiber Biosensors for Detection of Biomolecules. Advanced materials (Deerfield Beach, Fla.) 26, 4954-4960

50. Forzani, E.S., et al. (2004) A Conducting Polymer Nanojunction Sensor for Glucose Detection. Nano Letters 4, 1785-1788

51. Rivnay, J., et al. (2015) High-performance transistors for bioelectronics through tuning of channel thickness. Science Advances 1

52. Strakosas, X., et al. (2015) The organic electrochemical transistor for biological applications. Journal of Applied Polymer Science 132, 12

53. Pappa, A.-M., et al. (2017) Polyelectrolyte Layer-by-Layer Assembly on Organic Electrochemical Transistors. ACS Applied Materials \& Interfaces 9, 10427-10434

54. N. Bartlett, P. (1998) Measurement of low glucose concentrations using a microelectrochemical enzyme transistor. Analyst 123, 387-392

55. Zhu, Z.-T., et al. (2004) A simple poly(3,4-ethylene dioxythiophene)/poly(styrene sulfonic acid) transistor for glucose sensing at neutral pH. Chemical Communications, 1556-1557

56. Khodagholy, D., et al. (2012) Organic electrochemical transistor incorporating an ionogel as a solid state electrolyte for lactate sensing. Journal of Materials Chemistry 22, 4440-4443

57. Zhang, M., et al. (2015) Highly sensitive glucose sensors based on enzyme-modified wholegraphene solution-gated transistors. Scientific Reports 5, 8311

58. Scheiblin, G., et al. (2015) Screen-printed organic electrochemical transistors for metabolite sensing. MRS Communications 5, 507-511

59. Mak, C.H., et al. (2015) Highly-sensitive epinephrine sensors based on organic electrochemical transistors with carbon nanomaterial modified gate electrodes. Journal of Materials Chemistry C 3 , 6532-6538

60. Krishnan, S., et al. (2008) Advances in polymers for anti-biofouling surfaces. Journal of Materials Chemistry 18, 3405-3413

61. Wei, F., et al. (2012) Serum Creatinine Detection by a Conducting-Polymer-Based Electrochemical Sensor To Identify Allograft Dysfunction. Analytical Chemistry 84, 7933-7937 
62. Liao, C., et al. (2015) Flexible organic electrochemical transistors for highly selective enzyme biosensors and used for saliva testing. Adv Mater 27, 676-681

63. Pappa, A.M., et al. (2016) Organic Transistor Arrays Integrated with Finger-Powered Microfluidics for Multianalyte Saliva Testing. Adv Healthc Mater 5, 2295-2302

64. Ji, X., et al. (2016) Highly Sensitive Metabolite Biosensor Based on Organic Electrochemical Transistor Integrated with Microfluidic Channel and Poly(N-vinyl-2-pyrrolidone)-Capped Platinum Nanoparticles. Advanced Materials Technologies 1, 1600042

65. Pavlou, A.K., et al. (2004) Detection of Mycobacterium tuberculosis (TB) in vitro and in situ using an electronic nose in combination with a neural network system. Biosensors \& bioelectronics 20, 538-544

66. Strand, N., et al. (2010) Chemically Polymerized Polypyrrole for On-Chip Concentration of Volatile Breath Metabolites. Sensors and actuators. B, Chemical 143, 516-523

67. Bihar, E., et al. (2016) A Disposable paper breathalyzer with an alcohol sensing organic electrochemical transistor. Scientific Reports 6, 27582

68. Larsen, S.T., et al. (2012) Characterization of poly(3,4-ethylenedioxythiophene):tosylate conductive polymer microelectrodes for transmitter detection. Analyst 137, 1831-1836

69. Strakosas, X., et al. (2017) Catalytically enhanced organic transistors for in vitro toxicology monitoring through hydrogel entrapment of enzymes. Journal of Applied Polymer Science 134

70. Braendlein, M., et al. (2017) Lactate Detection in Tumor Cell Cultures Using Organic Transistor Circuits. Advanced Materials, 1605744-n/a

71. Curto, V., et al. (2017) A multi-parametric organic transistor toolbox with integrated microfluidics for in line in vitro cell monitoring. Microsystems and Nonoengineering

72. Khan, Y., et al. (2016) Monitoring of Vital Signs with Flexible and Wearable Medical Devices. Advanced Materials 28, 4373-4395

73. Takamatsu, S., et al. (2015) Direct patterning of organic conductors on knitted textiles for longterm electrocardiography. 5, 15003

74. Bihar, E., et al. (2017) Fully Printed Electrodes on Stretchable Textiles for Long-Term Electrophysiology. Advanced Materials Technologies 2, 1600251

75. Gualandi, I., et al. (2016) Textile Organic Electrochemical Transistors as a Platform for Wearable Biosensors. 6, 33637

76. Heikenfeld, J. (2016) Technological leap for sweat sensing. Nature 529, 475-476

77. Parrilla, M., et al. (2016) A Textile-Based Stretchable Multi-Ion Potentiometric Sensor. Advanced Healthcare Materials 5, 996-1001 
78. Kim, D.H., et al. (2011) Epidermal Electronics. Science 333, 838-843

79. Jia, W.Z., et al. (2013) Electrochemical Tattoo Biosensors for Real-Time Noninvasive Lactate Monitoring in Human Perspiration. Analytical Chemistry 85, 6553-6560

80. Imani, S., et al. (2016) A wearable chemical-electrophysiological hybrid biosensing system for real-time health and fitness monitoring. Nature Communications 7

81. Pal, R.K., et al. (2016) Conducting polymer-silk biocomposites for flexible and biodegradable electrochemical sensors. Biosensors \& Bioelectronics 81, 294-302

82. Kurland, N.E., et al. (2013) Precise Patterning of Silk Microstructures Using Photolithography. Advanced Materials 25, 6207-6212

83. Kurland, N.E., et al. (2014) Silk Protein Lithography as a Route to Fabricate Sericin Microarchitectures. Advanced Materials 26, 4431

84. Graf, H. and Muhleman.Hr (1966) TELEMETRY OF PLAQUE PH FROM INTERDENTAL AREA. Helvetica Odontologica Acta 10, 94

85. Graf, H. and Muhlemann, H.R. (1969) ORAL TELEMETRY OF FLUORIDE ION ACTIVITY. Archives of Oral Biology 14, 259

86. Bandodkar, A.J. and Wang, J. (2014) Non-invasive wearable electrochemical sensors: a review. Trends in Biotechnology 32, 363-371

87. Kim, J., et al. (2015) Wearable salivary uric acid mouthguard biosensor with integrated wireless electronics. Biosensors \& Bioelectronics 74, 1061-1068

88. Shum, A.J., et al. (2009) Functional modular contact lens. In Biosensing li (Razeghi, M. and Mohseni, H., eds)

89. Parviz, B.A. (2009) FOR YOUR EYE ONLY. leee Spectrum 46, 36-41

90. Yao, H., et al. (2011) A Dual Microscale Glucose Sensor On A Contact Lens, Tested In Conditions Mimicking The Eye. In 2011 leee 24th International Conference on Micro Electro Mechanical Systems, pp. 25-28

91. Thomas, N., et al. (2012) A contact lens with an integrated lactate sensor. Sensors and Actuators B-Chemical 162, 128-134

92. Chu, M.X., et al. (2011) Soft contact lens biosensor for in situ monitoring of tear glucose as noninvasive blood sugar assessment. Talanta 83, 960-965

93. Lee, S., et al. (2017) Smart Contact Lenses with Graphene Coating for Electromagnetic Interference Shielding and Dehydration Protection. ACS Nano 
94. Tehrani, F., et al. (2015) Rapid Prototyping of a High Sensitivity Graphene Based Glucose Sensor Strip. PLOS ONE 10, e0145036

95. Shichiri, M., et al. (1982) WEARABLE ARTIFICIAL ENDOCRINE PANCREAS WITH NEEDLE-TYPE GLUCOSE SENSOR. Lancet 2, 1129-1131

96. Katz, E. (2014) Implantable Bioelectronics. John Wiley \& Sons

97. lost, R.M., et al. (2015) Glucose Biochip Based on Flexible Carbon Fiber Electrodes: In Vivo Diabetes Evaluation in Rats. Chemelectrochem 2, 518-521

98. Vaddiraju, S., et al. (2010) Emerging synergy between nanotechnology and implantable biosensors: A review. Biosensors \& Bioelectronics 25, 1553-1565 
a.

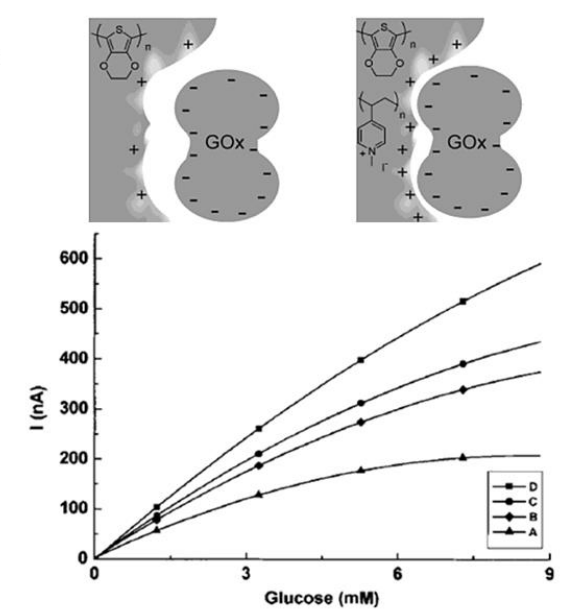

C.

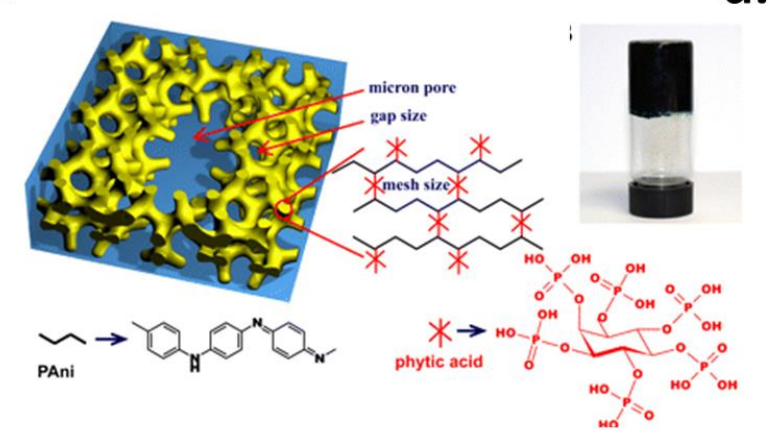

b.

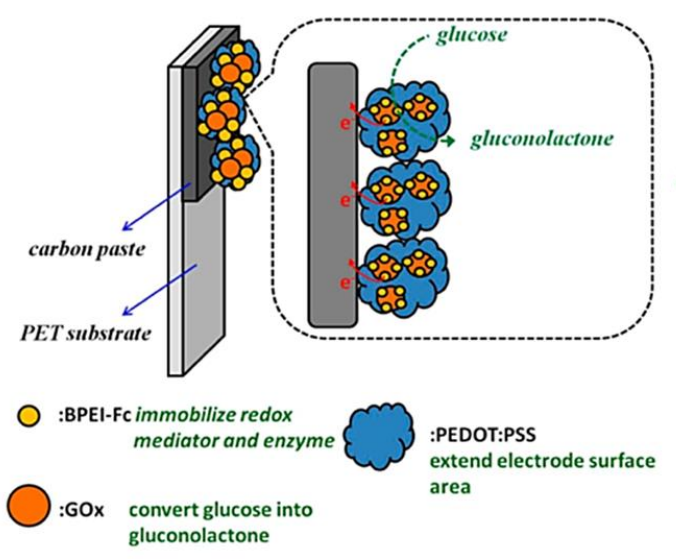

d.

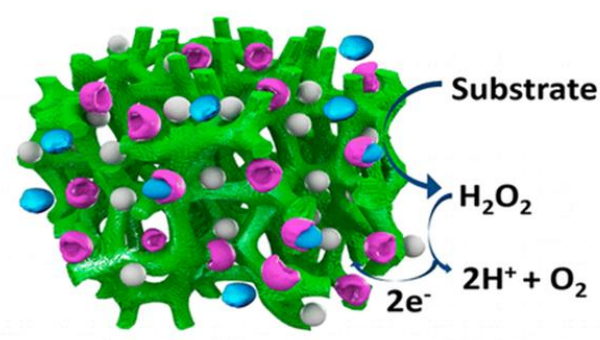

Figure 1: Organic electronic materials as efficient enzyme immobilization matrices and

transducers for metabolite detection. (a) Weak and strong electrostatic binding of glucose oxidase onto PEDOT matrix due to the positive charged PMVP inside the matrix and current response of the corresponding biosensor at $0.3 \mathrm{~V}$ vs $\mathrm{Ag} / \mathrm{AgCl}$ reference electrode as a function of glucose concentration for PEDOT only (A), as well as different PEDOT/PMVP molar ratio: 6(B), 3(C) and 2(D). Reproduced with permission from [41] (b) Schematic diagram of the working electrode coated with ferrocenebranched polyethylenimine, PEDOT:PSS and glucose oxidase for glucose detection with increased sensitivity Reproduced with permission from [43] (c) Chemical structure of phytic acid gelated and doped PANI hydrogel. Reproduced with permission from [45]. (d) Schematic of the PANI hydrogel matrix integrating platinum nanoparticles and the corresponding enzymes for the detection of uric acid, cholesterol and triglyceride. Reproduced with permission from [46] 


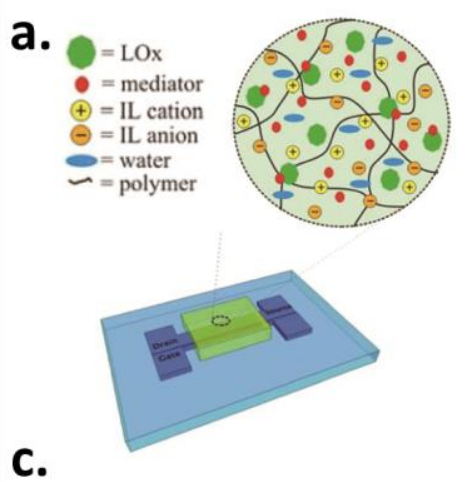

C.

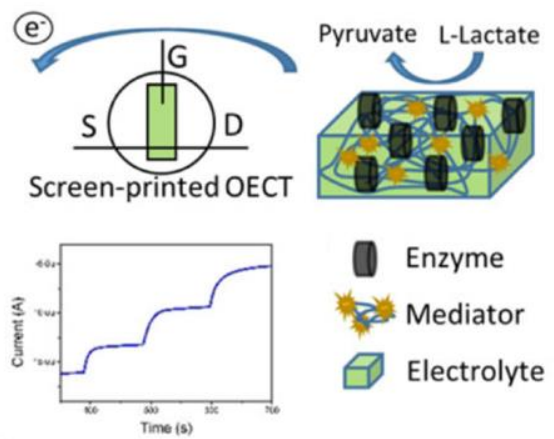

b.

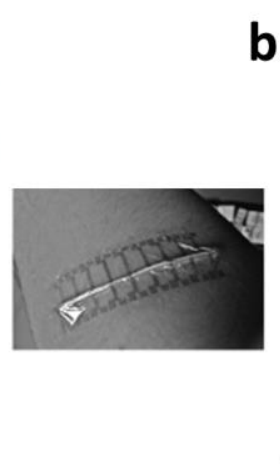

d.
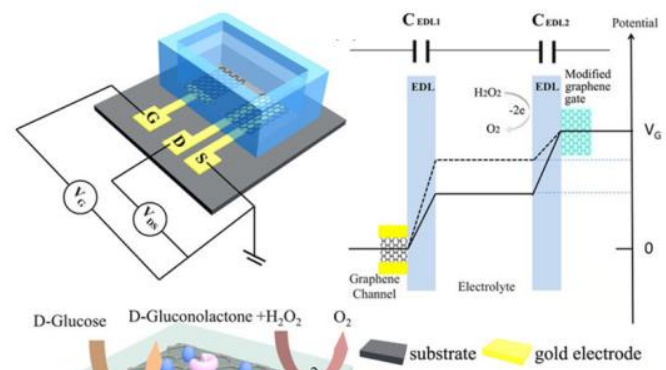

PDMS graphene

Pt nanoparticles polymer matrix

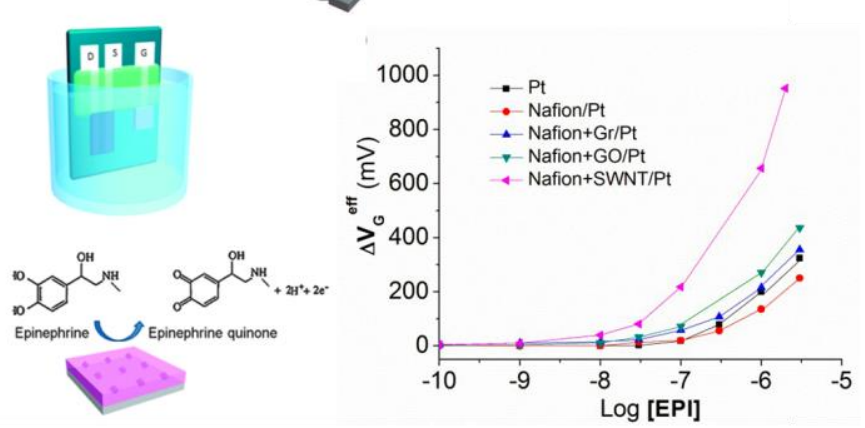

Figure 2. Highly integrated electronic devices bearing organic materials as the transducing elements for metabolite detection. (a) Schematic representation of the lactate OECT with an ionic gel solid state electrolyte, photograph of the actual flexible device and current modulation upon addition of lactate. Reproduced with permission from [56] (b) Illustration of the whole graphene solution gated transistor, showing the sensing electrode (gate) functionalization. Reproduced with permission from [57] (c) Schematic diagram of the screen printed OECT and illustration showing the gate electrode functionalization, comprised of a mediator as well as current response for the enzymatic determination of lactate. Reproduced with permission from [58] (d) Schematic representation of the epinephrine OECT based sensor bearing functionalized gate electrodes and potential drop at the gate electrode upon different concentrations of epinephrine dependent on the different functionalization schemes. Reproduced with permission from [59] 
a.



C.
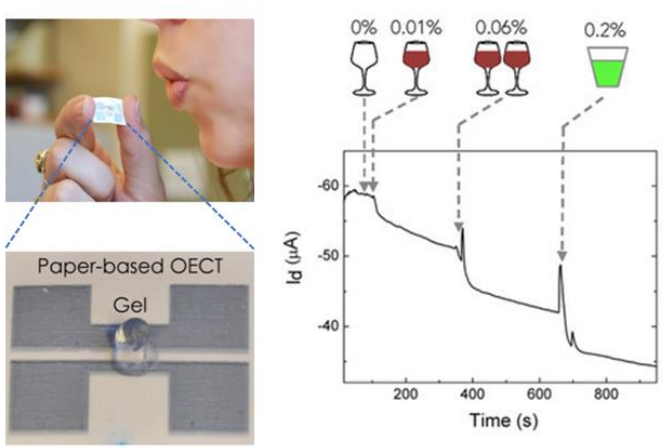

b.
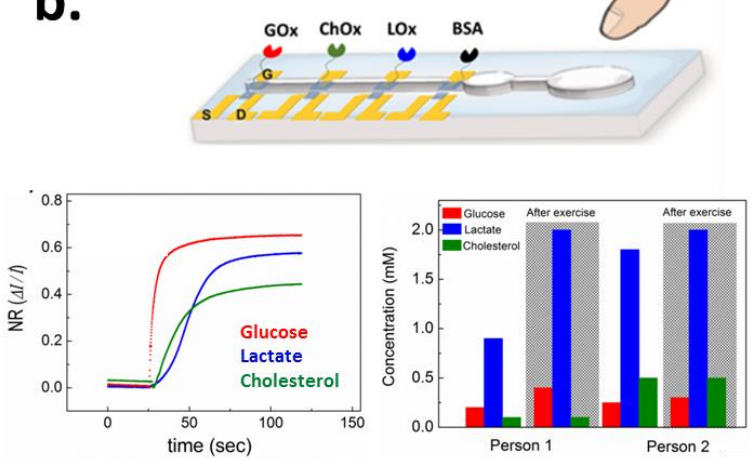

d.
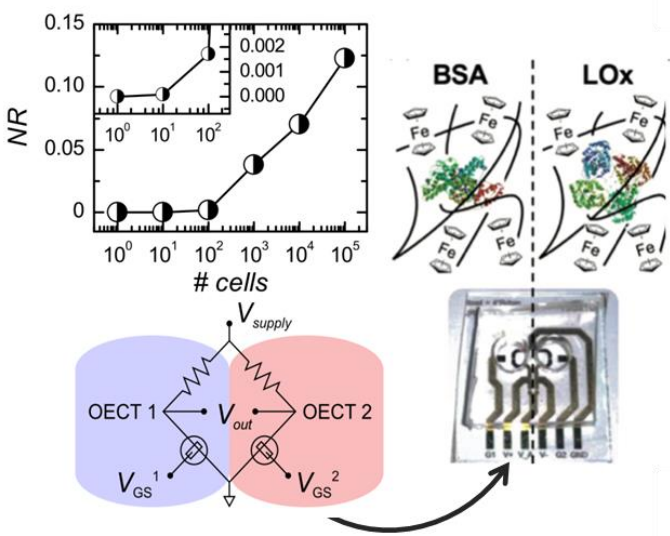

Figure 3. Fully integrated point-of-care platforms based on organic electronics for in vitro detection of metabolites and used in real world applications. (a) Illustration of the CP electrochemical sensor array for the direct measurement of creatinine from serum Reproduced with permission from [61] (b) Schematic showing the OECT-based multianalyte platform, simultaneous measurement of the three metabolites and the metabolites' levels of two healthy volunteers before and after exercise as measured by the proposed device. Reproduced with permission from [63] Photograph of the paper OECT- breathalyzer and curve showing the detection sensitivity. Reproduced with permission from [67] (d) Schematic of the sensitive reference-based OECT lactate sensing platform and lactate titration curve in different amounts of cells. Reproduced with permission from [70] 

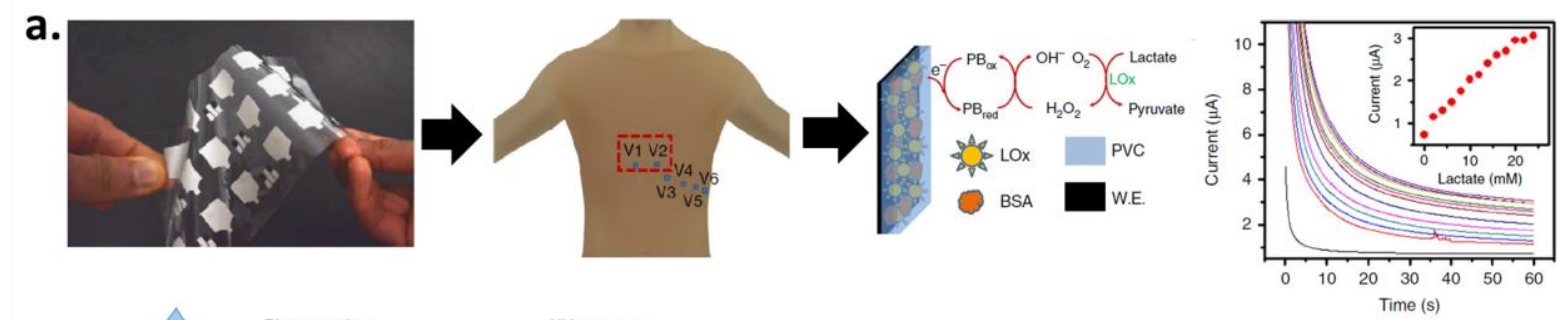

b.
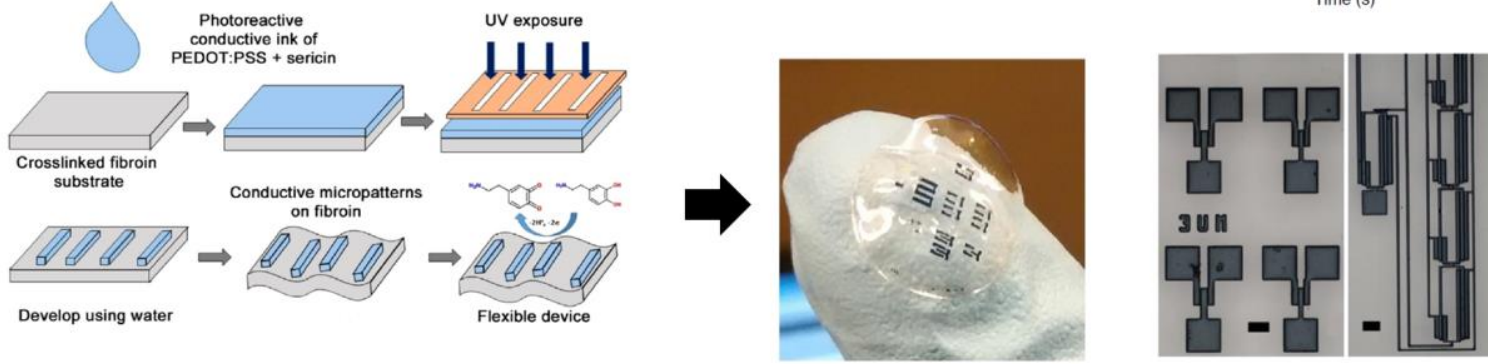

c.

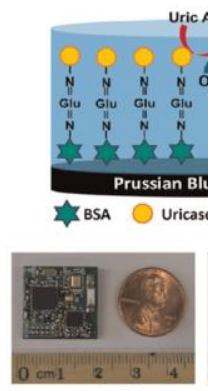

Front side
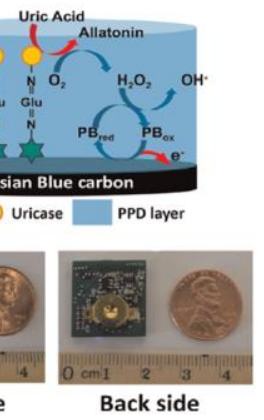

Back side

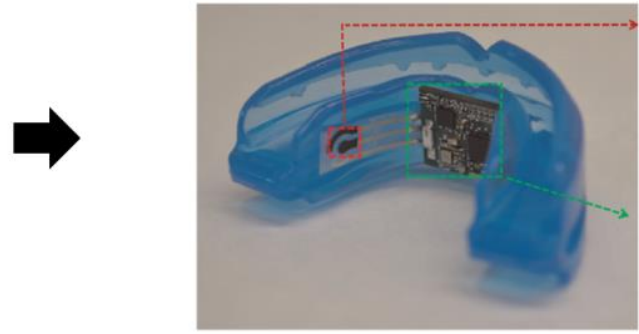

Figure 4. Non-invasive on body metabolite sensors based on organic materials. (a) Schematics of screen printed electrode, enzymatic sensing mechanism and amperometric response of wearable sweat-based lactate biosensor. Reproduced with permission from [80] (b) Schematic of fabrication steps on conductive micropattern on a flexible substrate, and large area micro patterns of flexible PEDOT:PSS on silk fibroin sheets with optical micrograph (Scalebars: $100 \mu \mathrm{m}$ ). Reproduced with permission from [81]. (c) Saliva-based mouthguard uric acid biosensor and its various components with sensing principles. Reproduced with permission from [87] 
a. fabrication of flexible electrode

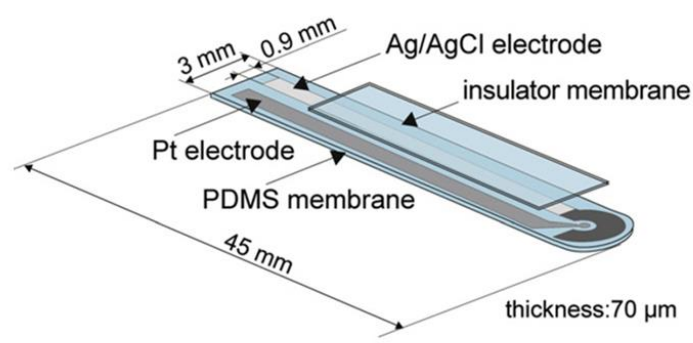

C.

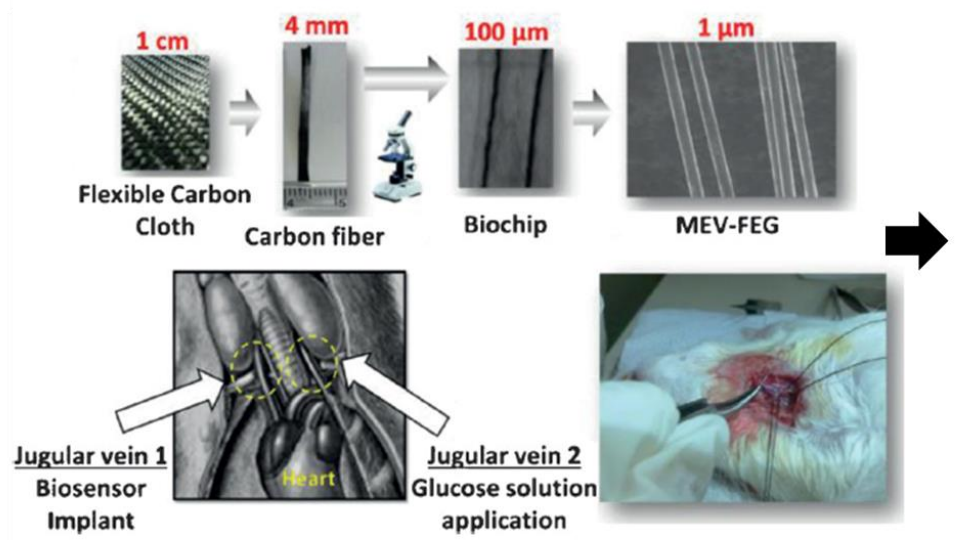

b. contact lens-type glucose sensor

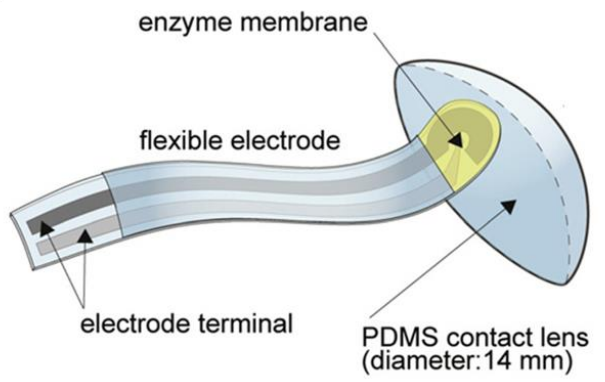

Figure 5. Minimally invasive and implantable metabolite sensors (a) The fabrication method of the contact lens biosensor on a flexible electrode and (b) integration of this electrode to the contact lens. Reproduced with permission from [92] (c) Images showing the device fabrication steps and implantation in a rat vein, and in vivo chronoamperometry measurements with Glucose injection after 20 min (zoomed region). Reproduced with permission from [97] 